\title{
ANTIOXIDANTES E BETA-GLUCANAS EM BARRAS DE CEREAIS COM Agaricus brasiliensis
}

\author{
KATIELLE R. VONCIK CÓRDOVA * \\ HERTA STUTZ DALLA SANTA ** \\ OSMAR ROBERTO DALLA SANTA *** \\ ELISA PEREZ * \\ NINA WASZCZYNSKYJ ${ }^{* * * * *}$
}

\begin{abstract}
O objetivo deste trabalho foi avaliar a capacidade antioxidante, a concentração de compostos fenólicos e de beta-glucanas em barras de cereais com Agaricus brasiliensis. As barras de cereais foram elaboradas por delineamento para mistura simplex-centroide para três variáveis: aveia, gergelim e trigo fermentado com $A$. brasiliensis. Os teores de $\beta$-glucanas nas amostras variaram de 1,30 a 3,82 g.100 g-1 e os compostos fenólicos de 67,45 a $81,96 \mathrm{mg}$ EAG. $100 \mathrm{~g}^{-1}$, constatando-se capacidade antioxidante na faixa de 29,47 a 40,17 mg CAET.100 g $\mathrm{g}^{-1}$. A adição de micélio de Agaricus brasiliensis em produtos alimentícios pode torná-los mais saudáveis, devido às propriedades nutritivas e compostos bioativos desse micro-organismo.
\end{abstract}

PALAVRAS-CHAVE: COMPOSTOS FENÓLICOS; GRÃOS DE TRIGO; FERMENTAÇÃO; TROLOX; ÁCIDO GÁLICO; POLISSACARÍDEOS.

* Doutoranda, Programa de Pós-Graduação em Engenharia de Alimentos, Universidade Federal do Paraná (UFPR), Docente, Curso de Engenharia de Alimentos, Universidade Estadual do Centro-Oeste (UNICENTRO), Guarapuava, PR (e-mail: kvcordova@hotmail.com).

** Doutora em Processos Biotecnológicos, Docente, Curso de Engenharia de Alimentos, UNICENTRO, Guarapuava, PR (e-mail: hdalsanta@yahoo.com.br).

*** Doutor em Tecnologia de Alimentos, Docente, Curso de Engenharia de Alimentos, UNICENTRO, Guarapuava, PR (e-mail: ordallasanta@yahoo.com.br).

**** Doutora em Química, Docente, Curso de Farmácia, UNICENTRO, Guarapuava, PR (e-mail: eperez@ unicentro.br).

***** Doutora em Ciências (Bioquímica), Docente Sênior, Programa de Pós-Graduação em Engenharia de Alimentos, UFPR, Curitiba, PR (e-mail: ninawas@ufpr.br). 


\section{INTRODUÇÃo}

O mercado dos produtos derivados de cogumelos medicinais encontra-se em ampla expansão. Estima-se que o mercado dos derivados de cogumelos medicinais e seus suplementos dietéticos, na última década, contribuiu com seis bilhões de dólares para a economia mundial. Assim, esse ramo tende a ser explorado cada vez mais (PAPASPYRIDI et al., 2011a).

O cogumelo Agaricus brasiliensis tem sido utilizado na prevenção de doenças coronarianas, de câncer e de diabetes, entre outras enfermidades (MIZUNO, 1999). Além de propriedades imunomoduladoras, apresenta substâncias efetivas para a diminuição do colesterol que ocasionam melhoria na hiperlipidemia, reduzem a pressão sanguínea e exercem atividades hipoglicêmica e antioxidante (DALLA SANTA et al., 2010).

As barras de cereais já fazem parte da dieta usual da população. Buscando alternativas que possibilitem a utilização de ingredientes mais saudáveis para a elaboração de barras de cereais, diversas pesquisas vêm sendo realizadas visando o desenvolvimento desses produtos com novos ingredientes alimentícios, nutritivos e/ou funcionais.

O consumo regular de beta-glucanas está relacionado à atenuação da resposta glicêmica e insulínica pós-prandial. Esse polissacarídeo exerce efeito na degradação do amido e do carboidrato disponível e, consequentemente, sobre o índice glicêmico dos alimentos ingeridos. Assim, recomenda-se a ingestão de beta-glucana com o objetivo de modular a glicemia e a necessidade de insulina (MIRA, GRAF e CÂNDIDO, 2009). Camelini et al. (2005), Ohno et al. (2001) e Itoh, Ito e Amano (1994) relataram que as $\beta$-glucanas dispõem de elevada atividade anti-tumoral, e por isso podem contribuir de forma importante na elaboração de produtos funcionais.

Biologicamente, antioxidantes podem ser definidos como compostos que protegem os sistemas biológicos contra os efeitos deletérios dos processos ou das reações que levam à oxidação de macromoléculas ou de estruturas celulares. Desta forma, os antioxidantes podem reduzir a incidência de diversas doenças (MOURÃO et al., 2011).

Os compostos fenólicos, substâncias antioxidantes, tem como principal função a proteção dos sistemas biológicos contra os defeitos degradantes dos processos e das reações que causam oxidação das moléculas ou estruturas celulares (VANNUCCHI e MARCHINI, 2007). Exibem grande quantidade de propriedades fisiológicas, mas o principal efeito dos compostos fenólicos tem sido atribuído à sua ação antioxidante em alimentos (ANDREO e JORGE, 2006).

O objetivo deste estudo foi avaliar a capacidade antioxidante, a concentração de compostos fenólicos e de beta-glucanas em barras de cereais adicionadas de trigo fermentado com Agaricus brasiliensis.

\section{MATERIAL E MÉTODOS}

\subsection{MATERIAL}

Para a elaboração da formulação padrão das barras de cereais foram utilizados os seguintes ingredientes: flocos de arroz tipo krispis, aveia em flocos, gergelim branco, coco ralado, gordura vegetal, lecitina de soja, xarope de glicose, açúcar mascavo e sal (LIMA et al., 2010; QUEIROZ et al., 2012; ROBERTO, 2012). Para as demais formulações, acrescentou-se o trigo fermentado com Agaricus brasiliensis.

Obteve-se o trigo fermentado mediante cultivo em estado sólido de grãos de trigo, previamente esterilizados e inoculados com Agaricus brasiliensis de cepa comercial (DALLA SANTA et al., 2010). Os grãos de trigo foram doados pela Cooperativa Agrária Agroindustrial de Entre Rios, Guarapuava (PR). 


\subsection{MÉTODOS}

\subsubsection{Obtenção do trigo fermentado}

Preparou-se o inóculo de $A$. brasiliensis em frascos Erlenmeyer de $1 \mathrm{~L}$, com $500 \mathrm{~mL}$ de meio estéril composto por (g. $\left.\mathrm{L}^{-1}\right)$ glicose (20), extrato de levedura (3,95), $\mathrm{MgSO}_{4} \cdot 7 \mathrm{H}_{2} \mathrm{O}(0,3) \mathrm{e} \mathrm{K}_{2} \mathrm{HPO}_{4} \cdot 3 \mathrm{H}_{2} \mathrm{O}$ $(0,5)$, sendo o pH ajustado a 6,0 $( \pm 0,2)$ em potenciômetro com $\mathrm{NaOH}(0,1 \mathrm{M})$. Inoculou-se o meio em câmara de fluxo laminar, coforme metodologia empregada por Dalla Santa et al. (2010), com incubação de sete dias e temperatura de $30^{\circ} \mathrm{C}$, sob agitação de $120 \mathrm{rpm}$. A suspensão de micélio de $A$. brasiliensis foi obtida por filtração em tela (malha de $0,5 \mathrm{~mm}^{2}$ ), utilizando-se $500 \mathrm{~mL}$ de água estéril destilada para lavagem da biomassa. $\mathrm{O}$ micélio foi gentilmente quebrado com espátula e passado por peneira, com auxílio de água destilada estéril (na proporção de 1:1 água destilada estéril para cultivo submerso), a fim de se obter suspensão de micélio quebrada (DALLA SANTA et al., 2010) a ser utilizada na inoculação do cultivo em estado sólido.

Realizou-se o cultivo em estado sólido para $A$. brasiliensis com grãos de trigo inteiros. Os grãos foram lavados e deixados de molho em água limpa por 12 horas para absorção da água. Após escoamento do excesso de água, acondicionou-se o substrato em embalagens de polipropileno autoclaváveis, adicionadas de quadrado de espuma na parte superior, amarrado com elástico de borracha, sendo cobertas com papel Kraft e esterilizadas a $121^{\circ} \mathrm{C}$ (pressão de $1 \mathrm{~atm}$ ), durante 45 min em autoclave. Depois do processo de esterilização, as embalagens de polipropileno foram colocadas em estufa a $100^{\circ} \mathrm{C}$ para secagem do papel a fim de se evitar contaminações e, em seguida, resfriadas à temperatura ambiente $\left(25 \pm 1^{\circ} \mathrm{C}\right)$, conforme Dalla Santa et al. (2012). A inoculação ocorreu em câmara de fluxo laminar com o inóculo na concentração de $5 \%(\mathrm{v} / \mathrm{m})$ de micélio por $\mathrm{Kg}$ de trigo. Efetuou-se o cultivo em estado sólido em temperatura de $30^{\circ} \mathrm{C}$, durante 21 dias em estufa com temperatura controlada.

O produto resultante do cultivo foi seco em estufa com circulação de ar e temperatura controlada de $55^{\circ} \mathrm{C}$ por 24 horas, sendo triturado em liquidificador industrial para obtenção da farinha contendo o micélio. Essa farinha passou por peneiramento em sistema vibratório de peneiras com granulometria $<0,5 \mathrm{~mm}$.

\subsubsection{Elaboração das barras de cereais}

O processo de elaboração das barras de cereais consistiu na caramelização da aveia com $50 \%$ do açúcar mascavo; aquecimento da calda aglutinante formada por xarope de glicose, $50 \%$ do açúcar mascavo, lecitina de soja e gordura vegetal; mistura dos ingredientes secos; mistura da calda aglutinante, da aveia caramelizada e dos ingredientes secos. Efetuou-se a laminação/ modelagem do produto usando moldes comerciais para barras de cereais, seguida por resfriamento durante duas horas. Padronizou-se o corte das barras no seguinte formato: comprimento de $900 \mathrm{~mm}$, largura de $300 \mathrm{~mm}$ e altura de $150 \mathrm{~mm}$. Após o corte, as barras de cereais foram imediatamente embaladas em papel alumínio e armazenadas em local seco e arejado. Cada barra de cereal pesou, em média, $25 \mathrm{~g} \pm 0,108$.

Para a elaboração das barras de cereais com trigo fermentado com A. brasiliensis utilizouse delineamento simplex-centroide para mistura de três componentes (DUTCOSKY et al., 2006), aumentado de pontos, totalizando 10 ensaios (Tabela 1). As variáveis estudadas incluíram as concentrações de aveia em flocos (AV), gergelim branco (GE) e trigo fermentado com A. brasiliensis (TR), estipulando-se $20 \%$ como nível máximo de cada variável e zero como mínimo (ausência). A aveia e o gergelim foram escolhidos como variáveis de estudo em testes preliminares devido às características sensoriais proporcionadas por esses ingredientes. A formulação controle (amostra 11), elaborada sem o trigo fermentado com A. brasiliensis, continha $16,3 \%$ de aveia em flocos e $3,7 \%$ de gergelim branco. 


\section{TABELA 1 - DELINEAMENTO EXPERIMENTAL PARA MISTURAS SIMPLEX-CENTROIDE PARA AS VARIÁVEIS AVEIA, GERGELIM E TRIGO FERMENTADO COM Agaricus brasiliensis}

\begin{tabular}{ccccccc}
\hline \multirow{2}{*}{ Experimento } & \multicolumn{3}{c}{ Níveis codificados } & \multicolumn{3}{c}{ Níveis originais (\%) } \\
& $\mathrm{x}_{1}$ & $\mathrm{x}_{2}$ & $\mathrm{x}_{3}$ & $\mathrm{AV}$ & $\mathrm{GE}$ & $\mathrm{TR}$ \\
\hline 1 & 1,000 & 0 & 0 & 20,000 & 0 & 0 \\
2 & 0 & 1,000 & 0 & 0 & 20,000 & 0 \\
3 & 0 & 0 & 1,000 & 0 & 0 & 20,000 \\
4 & 0,500 & 0,5 & 0 & 10,000 & 10,000 & 0 \\
5 & 0,500 & 0 & 0,500 & 10,000 & 0 & 10,000 \\
6 & 0 & 0,500 & 0,500 & 0 & 10,000 & 10,000 \\
7 & 0,333 & 0,333 & 0,333 & 6,666 & 6,666 & 6,666 \\
8 & 0,666 & 0,166 & 0,166 & 13,333 & 3,333 & 3,333 \\
9 & 0,166 & 0,666 & 0,166 & 3,333 & 13,333 & 3,333 \\
10 & 0,166 & 0,166 & 0,666 & 3,333 & 3,333 & 13,333 \\
\hline
\end{tabular}

Nota: AV (aveia), GE (gergelim), TR (trigo fermentado com A. brasiliensis).

\subsubsection{Determinação de Beta-Glucanas}

A determinação de beta-glucanas foi realizada em triplicata, conforme metodologia descrita por Papaspyridi et al. (2011 b) e Park et al. (2003), que consiste em hidrólises enzimáticas e químicas.

Determinou-se a porcentagem de $\beta$-glucanas quantitativamente, usando kit para determinação de $\beta$-glucanas para cogumelos e leveduras K-YBGL 09/2009 da Megazyme (Megazyme Internacional Ireland Ltd., Wicklow, UK) de acordo com as instruções do fabricante (LEE et al., 2011).

Para determinação das glucanas totais, as amostras de trigo in natura, trigo fermentado e barras de cereais foram trituradas, pesadas $(0,1 \mathrm{~g} \pm 0,01)$ e passadas em peneiras vibratórias - mesh $<0,5 \mathrm{~mm}(\mathrm{n}=3)$. A cada amostra pesada, uma amostra par foi armazenada para a determinação de umidade. As amostras foram hidrolisadas com $1,3 \mathrm{M}$ de $\mathrm{HCl}$ a $100^{\circ} \mathrm{C}$, durante $2 \mathrm{~h}$, sendo o $\mathrm{pH}$ neutralizado com $2 \mathrm{M}$ de $\mathrm{KOH}$. Para determinação das glucanas totais ( $\alpha$ e $\beta$ ) digeriuse uma alíquota de $0,1 \mathrm{~mL}$ com exo-1,3- $\beta$-glucanase $\left(100 \mathrm{U} \cdot \mathrm{mL}^{-1}\right)$ e $\beta$-glucosidase $\left(20 \mathrm{U} \cdot \mathrm{mL}^{-1}\right)$ em $200 \mathrm{mM}$ de tampão de acetato de sódio ( $\mathrm{pH} \mathrm{5,0).} \mathrm{Os} \mathrm{hidrolisados} \mathrm{foram} \mathrm{incubados} \mathrm{com} \mathrm{mistura} \mathrm{de}$ invertase $\left(500 \mathrm{U} \cdot \mathrm{mL}^{-1}\right)$, glicose oxidase $\left(>12.000 \mathrm{U} \cdot \mathrm{L}^{-1}\right)$, a $40^{\circ} \mathrm{C}$ durante $1 \mathrm{~h}$. Mediu-se a absorbância da solução em quintuplicata a $510 \mathrm{~nm}$ em espectrofotômetro Spectrumlab-22 PC.

As amostras foram dissolvidas em $2 \mathrm{M} \mathrm{KOH}$ e hidrolisadas, sendo adicionado 1,2 $\mathrm{M}$ de

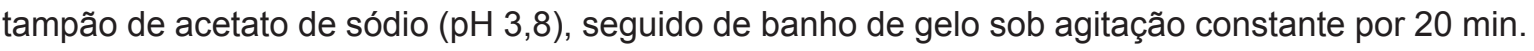
Amiloglucosidase (1630 U. $\left.\mathrm{mL}^{-1}\right)$ e invertase $\left(500 \mathrm{U} \cdot \mathrm{mL}^{-1}\right)$ foram adicionadas às amostras hidrolisadas e essas incubadas a $40^{\circ} \mathrm{C}$ durante $30 \mathrm{~min}$. Alíquotas da mistura de glicose oxidase (>12.000 U.L-1) e peroxidase (> 650 U.L-1 $)$ foram incubadas a $40^{\circ} \mathrm{C}$, durante $20 \mathrm{~min}$. Mediu-se a absorbância das amostras em quintuplicata a $510 \mathrm{~nm}$.

O conteúdo de beta-glucanas foi calculado pela diferença entre o conteúdo de glucanas totais e alfa-glucana (Equação 1): 


\subsubsection{Determinação de Antioxidantes}

\subsubsection{Extração etanólica}

Adaptou-se a metodologia utilizada para extração etanólica de Larrauri, Rupérez e SauraCalixto (1997). As amostras de trigo in natura, trigo fermentado e barras de cereais trituradas (granulometria inferior a 0,250 mm) foram pesadas $(0,1 \mathrm{~g}, \mathrm{n}=6)$ e extraídas com etanol $50 \% \mathrm{v} / \mathrm{v}(1,5 \mathrm{~mL})$ por 5 min em vórtex. Uma amostra de cada pesagem foi armazenada para a determinação de umidade.

A mistura (amostra + etanol) foi centrifugada (19975 x g, $15 \mathrm{~min}$ ) em microcentrífuga refrigerada, sendo o sobrenadante recolhido em balão volumétrico. A extração foi repetida, com o sobrenadante adicionado ao mesmo balão, completando-se $5 \mathrm{~mL}$ com etanol.

\subsubsection{Determinação do resíduo seco}

A quantificação do resíduo seco dos extratos ocorreu por método gravimétrico (IAL, 2008). Cinco alíquotas ( $5 \mathrm{~mL}$ ) desses extratos foram colocadas em placas de Petri, devidamente higienizadas e pesadas, e submetidas a $40^{\circ} \mathrm{C}$ em estufa de secagem por $24 \mathrm{~h}$. As placas foram retiradas da estufa, resfriadas em dessecador e pesadas. As placas foram colocadas em estufa por mais $24 \mathrm{~h}$, repetindo-se o processo até peso constante, visando obtenção do resíduo seco em $\mathrm{mg} \cdot \mathrm{mL}^{-1}$ de extrato. Esses resultados foram usados para expressar o conteúdo de fenólicos totais e a atividade antioxidante em base seca.

\subsubsection{Capacidade antioxidante total}

Determinou-se a capacidade antioxidante total dos grãos de trigo, do trigo fermentado e das barras de cereais elaboradas. Essa análise foi realizada pelo método do fosfomolibdênio, que se baseia na determinação espectrofotométrica da redução do molibdênio $\mathrm{Mo}^{+4} \mathrm{a} \mathrm{Mo}^{+5}$, com a subsequente formação de fosfato de $\mathrm{Mo}^{+5}$ que apresenta absorção máxima a $695 \mathrm{~nm}$. Alíquotas de $0,1 \mathrm{~mL}$ dos extratos (10 mg.mL ${ }^{-1}$ ) dissolvidas em água destilada a $1 \%$ (frações) foram combinadas, em tubo Eppendorf, com $1 \mathrm{~mL}$ da solução reagente (ácido sulfúrico $600 \mathrm{mM}$, fosfato de sódio $28 \mathrm{mM}$ e molibdato de amônio $4 \mathrm{mM}$ ). Os tubos foram fechados e incubados a $95^{\circ} \mathrm{C}$ por $90 \mathrm{~min}$. Após resfriamento à temperatura ambiente $\left(25 \pm 1^{\circ} \mathrm{C}\right)$, determinou-se a absorbância dos extratos etanólicos a $695 \mathrm{~nm}$. Utilizou-se trolox (6-Hidroxi-2,5,7,8-tetrametilcromano-2-ácido carboxílico; composto análogo à vitamina $\mathrm{E}$ ) como padrão, sendo a capacidade antioxidante total expressa como capacidade antioxidante equivalente à do trolox (CAET) por $100 \mathrm{~g}$ de resíduo seco (YADAV et al., 2013; PRIETO, PINEDA e AGUILAR, 1999).

\subsubsection{Determinação de compostos fenólicos totais}

Determinou-se o conteúdo de polifenois totais pelo método de Folin-Ciocalteau (SINGLETON e ROSSI, 1965), adaptado por Soares et al. (2009).

Cada fração do extrato $(0,1 \mathrm{~mL})$ foi misturada com $8,4 \mathrm{~mL}$ de água destilada e $0,5 \mathrm{~mL}$ de reagente Folin-Ciocalteau $(0,25 \mathrm{M})$, seguida de agitação e repouso por 3 minutos para reagir. Adicionou-se $1 \mathrm{~mL}$ de carbonato de sódio $20 \%(\mathrm{~m} / \mathrm{v})$ ao extrato que foi novamente agitado e mantido em repouso por 1 hora para reagir. Mediu-se a absorbância dos extratos etanólicos a $720 \mathrm{~nm}$, sendo utilizado ácido gálico para a curva analítica $\left(n=5 ; 0-200 \mathrm{mg} \cdot \mathrm{L}^{-1} ; \mathrm{y}=0,0025454+0,00069727 \times \mathrm{e}\right.$ $\left.R^{2}=0,993\right)$. $O$ conteúdo de fenólicos totais foi expresso em mg equivalentes em ácido gálico (EAG) por $100 \mathrm{~g}$ de resíduo seco.

\section{RESULTADOS E DISCUSSÃO}

\subsection{DETERMINAÇÃO DE BETA-GLUCANAS}

Na Figura 1 estão ilustrados os resultados obtidos para a determinação de beta-glucanas do 
trigo in natura, do trigo fermentado com $A$. brasiliensis, e das barras de cereais com trigo fermentado com A. brasiliensis.

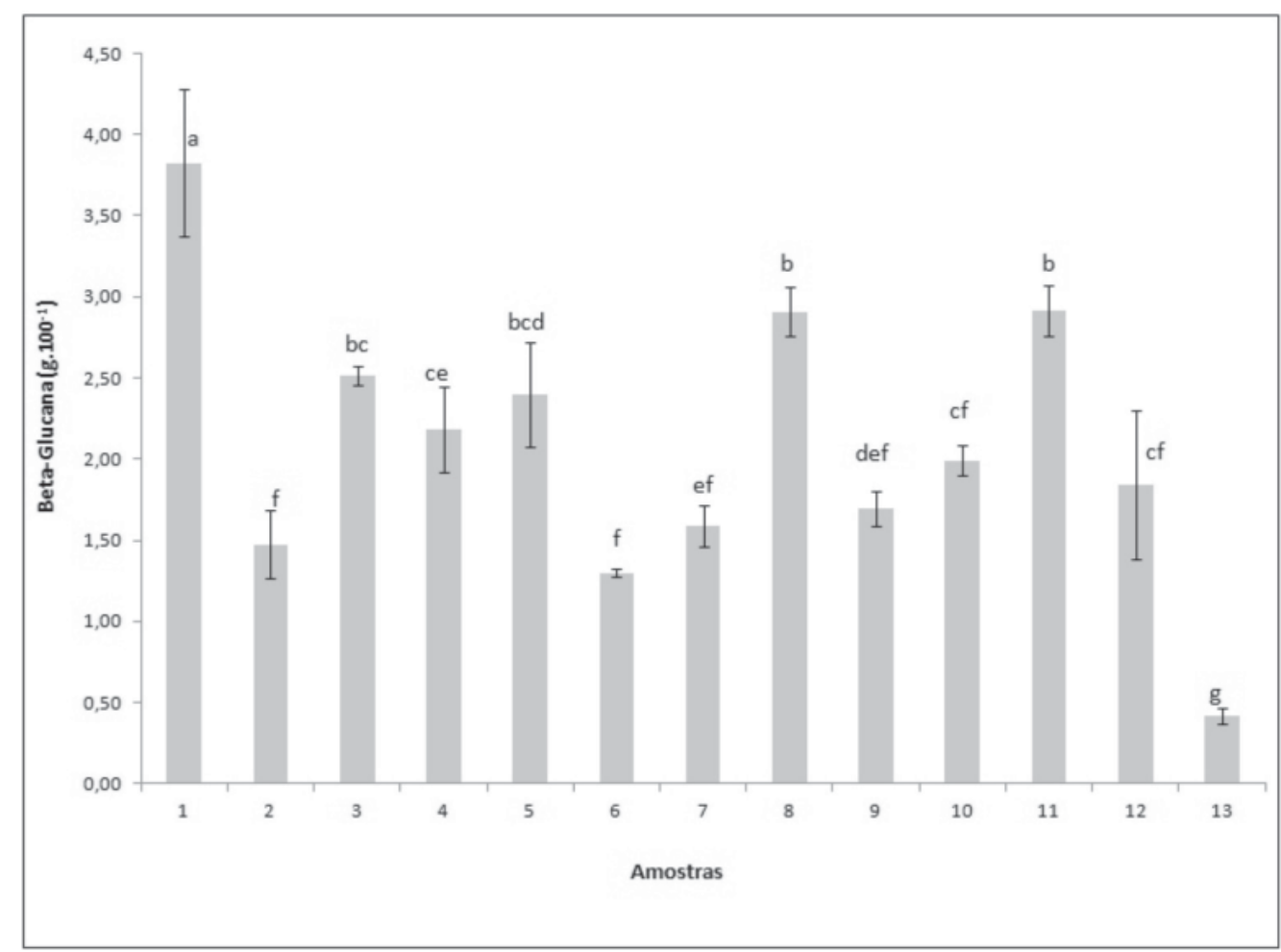

\section{FIGURA 1 - TEORES DE BETA-GLUCANAS DE TRIGO IN NATURA, TRIGO FERMENTADO COM Agaricus brasiliensis E DAS BARRAS DE CEREAIS COM Agaricus brasiliensis}

NOTA: Amostra 11 = controle; Amostra 12 = trigo fermentado com A. brasiliensis; Amostra $13=$ trigo in natura. Os resultados obtidos estão apresentados na forma de média \pm desvio padrão $(n=3)$. Letras iguais nas barras não diferem significativamente $(p<0,05)$.

Os teores de $\beta$-glucanas nas amostras de barras de cereais com A. brasiliensis variaram de 1,30 a 3,82 g. $100 \mathrm{~g}^{-1}$. A amostra 1 (20\% de aveia) diferiu significativamente das amostras 2 e 3, tendo a amostra 3 (20\% trigo fermentado com $A$. brasiliensis) evidenciado aumento significativo em relação à amostra 2 (20\% gergelim branco). Destaca-se tal amostra como alternativa interessante quando comparada a outros ingredientes habitualmente incorporados em barras de cereais, uma vez que os agáricos apresentam reconhecidas características nutricionais e medicinais (LOPES, SABAINI e GOMES-DA-COSTA, 2009).

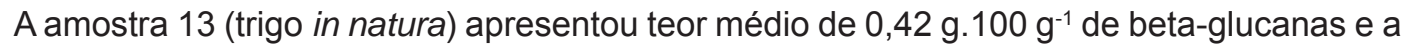
amostra 12 (trigo fermentado com $A$. brasiliensis) 1,84 g. $100 \mathrm{~g}^{-1}$. O valor médio de $\beta$-glucanas obtido para o trigo fermentado com $A$. brasiliensis foi 4,38 vezes superior ao resultado observado para 0 trigo in natura. $O$ teor de $\beta$-glucanas verificado para o trigo in natura mostrou-se próximo ao obtido por Fujita e Figueroa (2003), que relataram valores entre 0,47 a $0,72 \%$ em grãos de trigo. Não foram encontrados dados na literatura sobre beta-glucanas em trigo fermentado com fungos.

Gutcoski et al. (2007) determinaram $\beta$-glucanas em barras de cereais com aveia e observaram que as formulações contendo maiores teores de fibra alimentar também apresentaram os maiores teores de $\beta$-glucanas. De acordo com a legislação brasileira vigente, os produtos com o mínimo de $3 \mathrm{~g}$ de fibras ou de $\beta$-glucanas por $100 \mathrm{~g}$ podem ser considerados alimentos com "alto teor" de fibras e "fonte" de fibras, respectivamente. No presente estudo, apenas a amostra 1

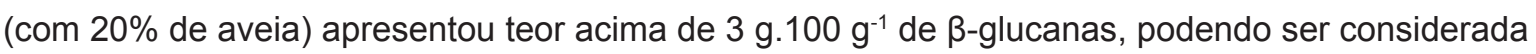
alimento funcional. A aveia tornou-se conhecida por sua alta concentração de beta-glucanas (BRASIL, 2002). 
As diferenças no teor de $\beta$-glucanas das barras de cereais podem ser explicadas pela variação dos níveis de aveia e trigo fermentado utilizado. Justifica-se o alto teor de $\beta$-glucanas (Figura 1) da amostra 1 pelo índice de aveia em flocos contido nessa formulação, considerando que o teor de $\beta$-glucanas nesse cereal varia de 4,50 a 6,50\% (FUJITA e FIGUEROA, 2003; GUTKOSKI e TROMBETTA, 1999). Saastamoinem, Plaami e Kumpulainem (1992) encontraram teores médios de 1,90 a $5,10 \%$ de $\beta$-glucanas em aveia. Cho e White (1993) relataram que o conteúdo de $\beta$-glucanas em grãos de 243 amostras de aveia mostrou distribuição normal, com a maioria das amostras apresentando em torno de 4,50 a $5,50 \%$ de $\beta$-glucanas por $100 \mathrm{~g}$ de aveia seca.

Shu, Lin e Wen (2007) encontraram valor máximo de $188 \mathrm{mg}^{-g^{-1}} \mathrm{de} \beta$-glucanas por biomassa de Agaricus blazei. Manzi e Pizzoferrato (2000) verificaram $\beta$-glucanas em Pleurotus ostreatus, $P$. pulmonarius, $P$. eryngii e Lentinula edodes, e constataram valores na faixa de 0,21 a $0,53 \mathrm{~g}$. $100 \mathrm{~g}^{-1}$ para cogumelo seco. Sandula et al. (1999) detectaram a presença de $\beta$-glucanas nas paredes celulares de Saccharomyces cerevisiae e de Aspergillus niger. Rosli e Aishah (2012) obtiveram em média 3,57 g.100 g $\mathrm{g}^{-1}$ de $\beta$-glucanas em Pleurotus sajor-caju (PSC).

\subsection{DETERMINAÇÃO DE ANTIOXIDANTES}

O valor médio do resíduo seco dos extratos de trigo in natura foi de $0,048 \pm 0,005 \mathrm{mg} \cdot \mathrm{mL}^{-1}$; para o trigo fermentado com $A$. brasiliensis de $0,049 \pm 0,006 \mathrm{mg} \mathrm{mL}^{-1}$; e para as barras de cereais de $0,047 \pm 0,005 \mathrm{mg} \cdot \mathrm{mL}^{-1}$.

Os resultados da avaliação da capacidade antioxidante total dos grãos de trigo, do trigo fermentado e das barras de cereais elaboradas estão ilustrados na Figura 2.

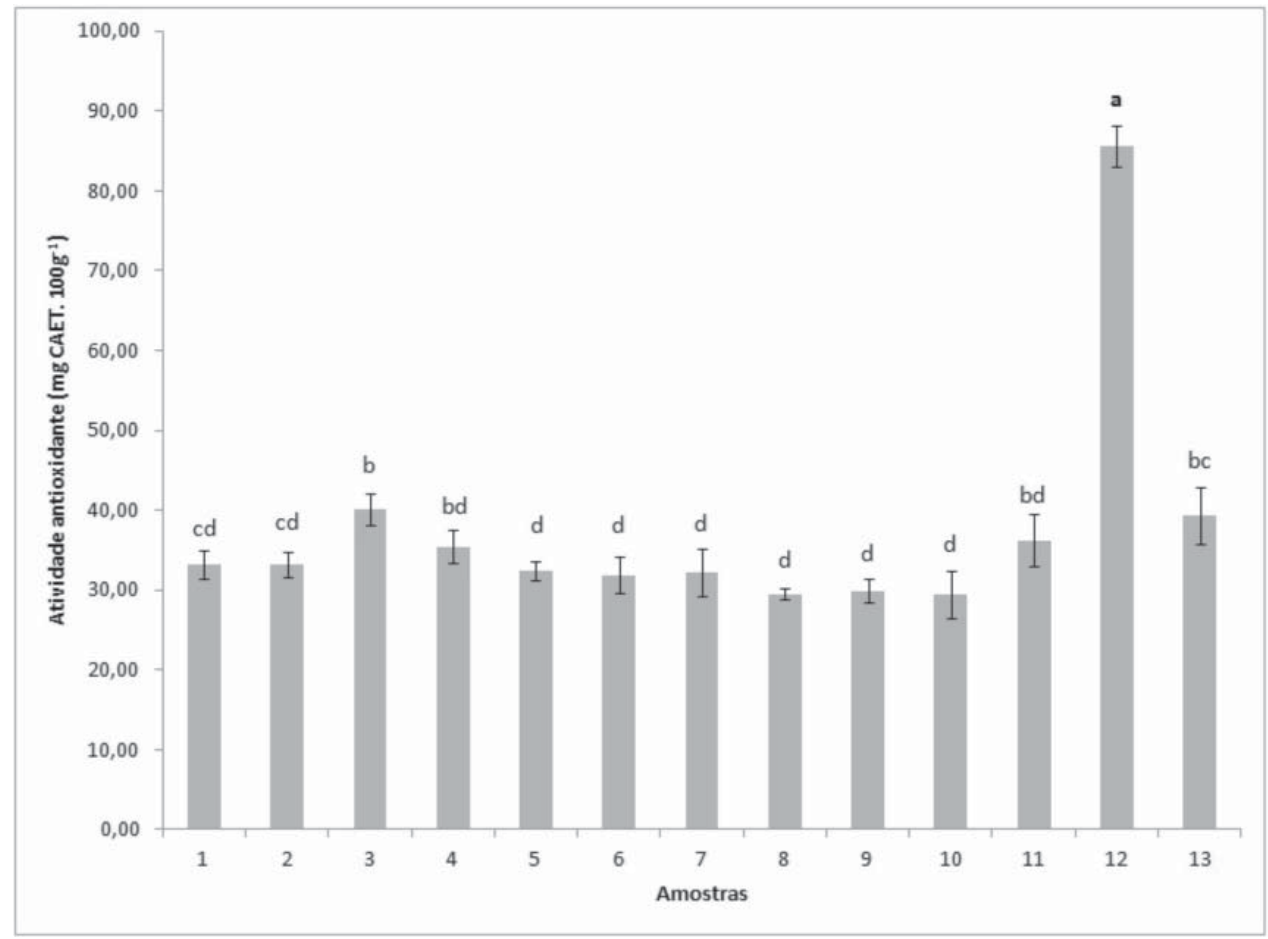

\section{FIGURA 2 - RESULTADOS DA AVALIAÇÃO DA CAPACIDADE ANTIOXIDANTE TOTAL DE TRIGO IN NATURA, TRIGO FERMENTADO COM Agaricus brasiliensis E DAS BARRAS DE CEREAIS COM Agaricus brasiliensis}

NOTA: resultados expressos em mg equivalentes de trolox (CAET) e mg por $100 \mathrm{~g}$ de resíduo seco. Amostra $11=$ controle; Amostra 12 = trigo fermentado com A. brasiliensis; Amostra $13=$ trigo in natura. Os resultados obtidos estão apresentados na forma de média \pm desvio padrão $(n=3)$. Letras iguais nas barras não diferem significativamente $(p<0,05)$. 
A capacidade antioxidante das amostras de barras de cereais com $A$. brasiliensis (1 a 11) variaram de 29,47 a 40,17 mg CAET.100 $\mathrm{g}^{-1}$, com diferença estatística $(p<0,05)$ entre si. As amostras de trigo fermentado (12) e trigo in natura (13) também diferiram significativamente, com elevação de aproximadamente 2,20 vezes nos teores de capacidade antioxidante do trigo

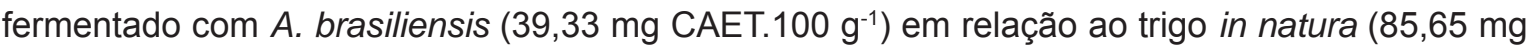
CAET.100 g $\left.{ }^{-1}\right)$.

A amostra 3 (Tabela 1) da barra de cereais com adição de $20 \%$ de trigo fermentado com A. brasiliensis diferiu estatisticamente das amostras com $20 \%$ de aveia (1) e $20 \%$ de gergelim (2), mas não da amostra $13 \mathrm{com}$ trigo in natura, embora com acréscimo de 2,13\% na capacidade antioxidante. A amostra de trigo fermentado com A. brasiliensis (12) evidenciou aumento próximo de $119 \%$ na capacidade antioxidante em relação à amostra 13 (trigo in natura). As amostras de 4 a 11, compostas por misturas das variáveis, não diferiram estatisticamente entre si.

Liyana-Pathirana e Shahidi (2007) avaliaram a capacidade antioxidante total de grãos de trigo inteiros e derivados, obtendo teor médio de 4,99 $\pm 0,06 \mu \mathrm{mol}$ de trolox. $\mathrm{g}^{-1}$ de trigo. AlvarezJubete et al. (2010) analisaram os antioxidantes de grãos de trigo e constataram teor médio de $44,1 \pm 0,4 \mathrm{mg}$ trolox. $100 \mathrm{~g}^{-1}$ de trigo, valor próximo ao verificado no presente estudo $(39,33 \mathrm{mg}$ CAET. $\left.100 \mathrm{~g}^{-1}\right)$.

Zhang etal. (2012) avaliaram a atividade antioxidante de trigo (Triticum aestivum Linn) e trigo fermentado em estado sólido com Cordyceps militaris. Obtiveram $64,52 \%$ de atividade antioxidante para o trigo sem o processo fermentativo e $71,21 \%$ para o trigo fermentado com concentração de $0,1 \mathrm{mg} \cdot \mathrm{mL}^{-1}$ de amostra, ou seja, aumento de aproximadamente $6,70 \%$ na atividade antioxidante após a fermentação. No presente estudo verificou-se aumento de $119 \%$ na capacidade antioxidante do trigo fermentado com $A$. brasiliensis em relação aos grãos de trigo in natura, o que evidencia o alto poder antioxidante desse cogumelo. Silva et al. (2009) também comprovaram a elevada capacidade antioxidante desse cogumelo $(28,90 \%)$, indicando que os agáricos constituem promissores agentes antioxidantes naturais.

Alezandro et al. (2011) avaliaram a atividade antioxidante de barras de cereais de soja com mel e obtiveram $20 \mathrm{mg}$ CAET. $100 \mathrm{~g}^{-1}$, valores inferiores aos constatados no presente trabalho (teor médio de 33,06 $\pm 3,24$ ).

A quantidade de antioxidantes de cada planta depende de diferentes fatores, incluindo as propriedades coloidais dos substratos, as condições e etapas de oxidação, a formação e a estabilidade dos radicais, assim como a provável localização dos antioxidantes e estabilidade em distintas fases do processamento dos alimentos (ROCKENBACH et al., 2007). As diferenças observadas na atividade antioxidante podem ser ainda maiores quando se analisa alimentos (matriz complexa), cujos componentes podem estabelecer inúmeras e diferentes interações entre si e com os solventes (ROCKENBACH et al., 2008).

Os resultados da determinação de compostos fenólicos totais dos grãos de trigo, de trigo fermentado e das barras de cereais elaboradas são apresentados na Figura 3.

Os resultados dos teores de compostos fenólicos (Figura 3) nas amostras de barras de cereais com $A$. brasiliensis variaram de 67,45 a 81,96 mg EAG.100 g-1. Não foi constatada diferença estatística $(p<0,05)$ entre as amostras 1 a 4 , assim como entre as amostras 5 a 11 , porém esses dois grupos diferiram entre si e também das amostras 12 e 13.

Moraes et al. (2007) elaboraram barras de cereais adicionadas de passas de mirtilo e verificaram teor médio de compostos fenólicos de $23,70 \pm 4,70 \mathrm{mg}$ de quercetina. $\mathrm{g}^{-1}$ de produto final. Sun-Waterhouse et al. (2010) desenvolveram barra de cereais funcional com adição de

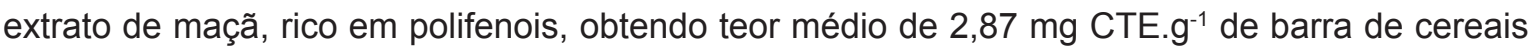
(CTE = equivalente catequina). 


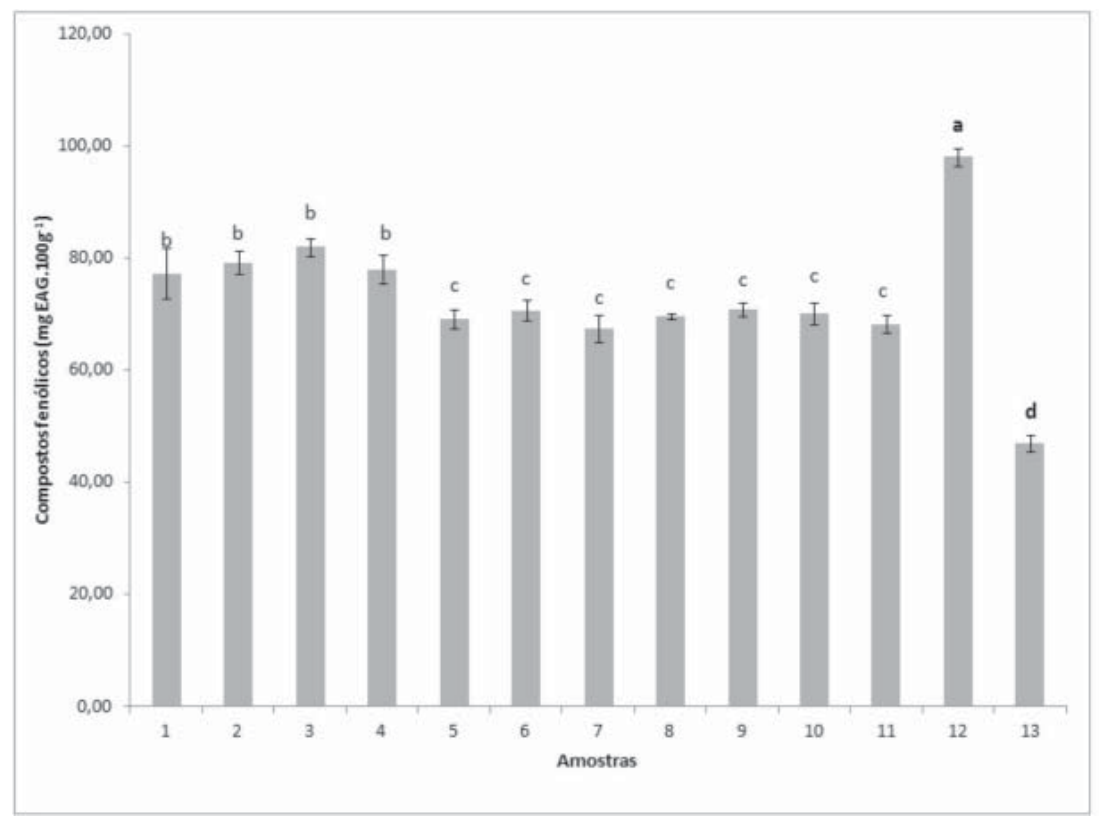

\section{FIGURA 3 - RESULTADOS DA DETERMINAÇÃO DOS COMPOSTOS FENÓLICOS TOTAIS DE TRIGO IN NATURA, TRIGO FERMENTADO COM Agaricus brasiliensis E DAS BARRAS DE CEREAIS COM Agaricus brasiliensis}

NOTA: resultados expressos em mg equivalentes de ácido gálico (EAG) por $100 \mathrm{~g}$ de resíduo seco. Amostra 11 = controle; Amostra 12 = trigo fermentado com A. brasiliensis; Amostra 13 = trigo in natura. Os resultados obtidos estão apresentados na forma de média \pm desvio padrão $(n=3)$. Letras iguais nas barras não diferem significativamente $(p<0,05)$.

As amostras de trigo fermentado (12) e de trigo in natura (13) diferiram entre si e das demais quanto aos teores de fenólicos totais (98,03 mg EAG.100 g $\mathrm{g}^{-1}$ e 47,03 mg EAG. $100 \mathrm{~g}^{-1}$, respectivamente). A amostra 12 (trigo fermentado com $A$. brasiliensis) mostrou aumento próximo a 108\% no teor de compostos fenólicos em relação à amostra 13 (trigo in natura), evidenciando a capacidade desse cogumelo em produzir moléculas bioativas. Silva e Jorge (2011), avaliando compostos fenólicos em corpos de frutificação de $A$. blazei, encontraram resultados na faixa de 26,67 a $28,89 \mathrm{mg}^{-\mathrm{g}^{-1}}$ de fenólicos totais, enquanto Soares et al. (2009) obtiveram de 28,82 a $29,64 \mathrm{mg} \cdot \mathrm{g}^{-1}$ de fenólicos totais para A. blazei em dois estádios de maturação do corpo de frutificação.

Liyana-Pathirana e Shahidi (2007) avaliaram o teor de compostos fenólicos de grãos de trigo inteiros e derivados, e obtiveram valor médio de $1291 \pm 31 \mu \mathrm{g}$ de FAE (equivalente ácido ferúlico) por grama de trigo. Alvarez-Jubete et al. (2010), analisando antioxidantes em grão de trigo, encontraram teor médio de fenólicos totais de 53,1 $\pm 2,8$ mg EAG. $100 \mathrm{~g}^{-1}$ de trigo. Os valores de fenólicos relatados por tais pesquisadores foram superiores aos verificados neste estudo, devendo-se considerar a variação de espécie, estresse ambiental a que a planta foi submetida, estágios de crescimento e maturação, além da metodologia analítica empregada para sua determinação (CRUZ et al., 2011).

O enriquecimento de alimentos com compostos fenólicos por meio de fermentação em estado sólido foi relatado para o feijão preto (LEE, HUNG e CHOU, 2008), a soja (LIN, WEI e CHOU, 2006) e o arroz (BHANJA et al., 2008). Liang, Syu e Mau (2009) utilizaram Phellinus linteus para fermentação em estado sólido de arroz e obtiveram valor médio de 4,38 $\pm 0,03 \mathrm{mg} \cdot \mathrm{g}^{-1}$ fenois totais (expressos em equivalente em ácido cafeico) para o extrato aquoso e de 4,54 $\pm 0,02 \mathrm{mg}^{-\mathrm{g}^{-1}}$ fenois totais para o extrato etanólico.

A presença de antioxidantes nas barras de cereais, em qualquer quantidade, representa diferencial sob o ponto de vista nutricional, uma vez que a legislação brasileira (BRASIL, 2003) não exige teor mínimo de antioxidantes no produto. Em vista disso, pode-se dizer que as barras de cereais formuladas nesse estudo podem gerar benefícios ao consumidor, pois possibilitam o enriquecimento de sua dieta com antioxidantes para combater o efeito oxidativo e deletério dos radicais livres. 


\section{CONCLUSÃO}

Os teores de $\beta$-glucanas nas amostras de barras de cereais com $A$. brasiliensis variaram

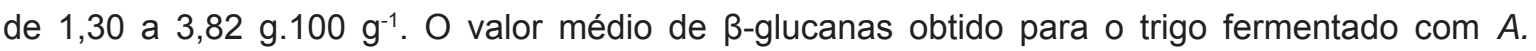
brasiliensis foi 4,38 vezes superior ao resultado observado para o trigo in natura. A amostra 1 (com

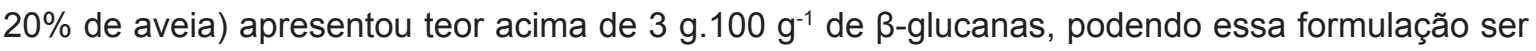
considerada como alimento funcional.

Acapacidade antioxidante das amostras de barras de cereais com $A$. brasiliensis variaram de 29,47 a 40,17 mg CAET.100 g-1. A amostra de trigo fermentado com A. brasiliensis mostrou aumento próximo de $119 \%$ na capacidade antioxidante quando comparada à amostra de trigo in natura.

As concentrações de compostos fenólicos nas amostras de barras de cereais com $A$. brasiliensis variaram de 67,45 a $81,96 \mathrm{mg}$ EAG. $100 \mathrm{~g}^{-1}$. O trigo fermentado revelou aumento próximo de $108 \%$ no teor de compostos fenólicos em relação ao trigo in natura.

A adição de micélio de Agaricus brasiliensis em produtos alimentícios pode torná-los mais saudáveis, devido às propriedades nutritivas e compostos bioativos desse micro-organismo.

\section{ABSTRACT}

\section{ANTIOXIDANTS AND BETA-GLUCAN IN CEREAL BARS WITH Agaricus brasiliensis}

The aim of this study was to evaluate the antioxidant capacity, phenolic compounds and beta-glucans from cereal bars with Agaricus brasiliensis. The cereal bars were prepared by mixing design for simplex-centroid for three variables: oats, sesame and fermented wheat with $A$. brasiliensis. The content of $\beta$-glucan varied from 1.30 to $3.82 \mathrm{~g} .100 \mathrm{~g}^{-1}$, phenolic compounds ranged from 67.45 to $81.96 \mathrm{mg}^{\mathrm{G} A E} .100 \mathrm{~g}^{-1}$ and showed antioxidant capacity in the range of 29.47 to $40.17 \mathrm{mg} \mathrm{TECA.100} \mathrm{g-}^{-1}$. The addition of mycelium of Agaricus brasiliensis in food can make them healthier due to its nutritional properties and bioactive compounds of this micro-organism.

KEY-WORDS: PHENOLIC COMPOUNDS; WHEAT GRAINS FERMENTATION; TROLOX; GALLIC ACID; POLYSACCHARIDES.

\section{REFERÊNCIAS}

1 ALEZANDRO, M. R.; GRANATO, D.; LAJOLO, F. M.; GENOVESE, M. I. Nutritional aspects of second generation soy foods. J. Agric. Food Chem., v. 59, 5490-5497, 2011.

2 ALVAREZ-JUBETE, L.; WIJNGAARD, H.; ARENDT, E. K.; GALLAGHER, E. Polyphenol composition and in vitro antioxidant activity of amaranth, quinoa buckwheat and wheat as affected by sprouting and baking. Food Chemistry, v. 119 , p. $770-778,2010$.

3 ANDREO, D.; JORGE, N. Antioxidantes naturais: técnicas de extração. B. do CEPPA, v. 24, n. 2, p. 319-336, 2006,

4 BHANJA, T.; ROUT, S.; BANERJEE, R.; BHATTACHARYYA, B. C. Studies on the performance of a new bioreactor for improving antioxidant potential of rice. LWT - Food Science and Technology, v. 42, p. 1459-1465, 2008.

5 BRASIL. Ministério da Saúde. Resolução ANVS/MS n. 2 de 07 de janeiro de 2002. Aprova regulamento técnico de substâncias bioativas e probióticos isolados com alegação de propriedades funcional e ou de saúde. Diário Oficial [da] República Federativa do Brasil, Brasília, 9 jan. 2002, Seção 1, p. 191.

6 BRASIL. Ministério da Saúde. Resolução ANVS/MS RDC n. 360, de 23 de dezembro de 2003. Aprova regulamento técnico sobre rotulagem nutricional de alimentos embalados, tornando obrigatória a rotulagem nutricional. Diário Oficial [da] República Federativa do Brasil. Brasília, 26 dez. 2003, Seção 1, p. 251.

7 CAMELINI, C. M.; MARASCHIN, M.; MENDONÇA, M. M.; ZUCCO, C.; FERREIRA, A. G.; TAVARES, L. A. Structural characterization of $\beta$-glucans of Agaricus brasiliensis in different stages of fruiting body maturity and their use in nutraceutical products. Biotechnology Letters, v. 27, p. 1295-1299, 2005.

$8 \mathrm{CHO}, \mathrm{K}$. C.; WHITE, P. J. Enzymatic analysis of beta-glucan content in different oat genotypes. Cereal Chemistry, v. 70 , n. 5, p. 539-542, 1993.

9 CRUZ, A. P. G.; MATTIETTO, R.; DIB TAXI, C.; CABRAL, L. M. C.; DONANGELO, C. M.; MATTA, V. M. Effect of microfiltration on bioactive components and antioxidant activity of açaí (Euterpe oleracea Mart.). Desalination and Water Treatment, v. 27, p. 97-102, 2011. 
10 DALLA SANTA, H. S.; RUBEL, R.; FERNANDES, L. C.; BONATTO, S. J. R.; BELLO, S. R.; MONTEIRO, M. C.; KHALIL, N. M.; DALLA SANTA, O. R.; SOCCOL, C. R.; GERN, J. C.; SANTOS, C. A. M. Agaricus brasiliensis-enriched functional product promotes in mice increase in HDL levels and immunomodulate to Th1 CD4+T subsets. A. brasiliensis functional product and biological benefits. Current Trends in Biotechnology and Pharmacy, v. 4, n. 4, p. 957-970, 2010.

11 DALLA SANTA, H. S.; RUBEL, R.; VITOLA, F. M. D.; RODRIGUEZ-LEON, J. A.; DALLA SANTA, O. R.; ALVARÉZ, D. C.; MACEDO, R. E. F.; CARVALHO, J. C.; SOCCOL, C. R. Growth parameters of Agaricus brasiliensis mycelium on wheat grains in solid-state fermentation. Biotechnology, v. 3, p. 144-153, 2012.

12 DUTCOSKY, S. D.; GROSSMANN, M. V. E.; SILVA, R. S. S. F.; WELSCH, A. K. Combined sensory optimization of a prebiotic cereal product using multicomponent mixture experiments. Food Chemistry, v. 98, p. 630-638, 2006.

13 FUJITA, A. H.; FIGUEROA, M. O. R. Composição centesimal e teor de $\beta$-glucanas em cereais e derivados. Ciênc. Tecnol. Aliment. , v. 23, n. 2, p. 116-120, 2003.

14 GUTKOSKI, L. C.; BONAMIGO, J. M. A.; TEIXEIRA, D M. F.; PEDÓ, I. Desenvolvimento de barras de cereais à base de aveia com alto teor de fibra alimentar. Ciênc. Tecnol. Aliment., v. 27, n. 2, p. 355-363, 2007.

15 GUTKOSKI, L. C.; TROMBETTA, C. Avaliação dos teores de fibra alimentar e de beta-glicanas em cultivares de aveia (Avena sativa L). Ciênc. Tecnol. Aliment., v. 19, n. 3, p. 387-390, 1999.

16 INSTITUTO ADOLFO LUTZ (IAL). Normas analíticas do Instituto Adolfo Lutz: métodos químicos e físicos para análise de alimentos. 4 ed. 1 ed. digital. São Paulo, 2008.

17 ITOH, H.; ITO, H.; AMANO, H. Inhibitory action of a (10/6)-b-D-glucan/protein complex (FIII-2-b) isolated from Agaricus blazei Murill ('Himematsutake') on Meth A fibrosarcomabearing mice and its antitumor mechanism. Japanese Journal of Pharmacology, v. 66, p. 265-271, 1994.

18 LARRAURI, J. A.; RUPÉREZ, P.; SAURA-CALIXTO, F. Effect of drying temperature on the stabilitity of polyphenols and antioxidant activity of red grape pomace peels. Journal Agriculture and Food Chemistry, v. 45, p. 1390-1393, 1997.

19 LEE, I.; HUNG, Y. H.; CHOU, C. Solid-state fermentation with fungi to enhance the antioxidative activity, total phenolic and anthocyanin contents of black bean. International Journal Food Microbiology, v. 121, p. 150-156, 2008.

20 LEE, J.; OKA, K.; WATANABE, O.; HARA, H.; ISHIZUKA, S. Immunomodulatory effect of mushrooms on cytotoxic activity and cytokine production of intestinal lamina propria leukocytes does not necessarily depend on b-glucan contents. Food Chemistry, v. 126, p. 1521-1526, 2011

21 LIANG, C.; SYU, J.; MAU, J. Antioxidant properties of solid-state fermented adlay and rice by Phellinus linteus. Food Chemistry, v. 116, p. 841-845, 2009.

22 LIMA, J. C.; FREITAS, J. B.; CZEDER, L. P.; FERNANDES, D. C.; NAVES, M. M. V. Qualidade microbiológica, aceitabilidade e valor nutricional de barras de cereais formuladas com polpa e amêndoa de baru. B. do CEPPA, v. 28, n. 2, p. 331-343, 2010.

23 LIN, C.; WEI, Y.; CHOU, C. Enhanced antioxidative activity of soybean koji prepared with various filamentous fungi. Food Microbiology, v. 23, p. 628-633, 2006.

24 LIYANA-PATHIRANA, C. M.; SHAHIDI, F. Antioxidant and free radical scavenging activities of whole wheat and milling fractions. Food Chemistry, v. 101, p. 1151-1157, 2007.

25 LOPES, A.; SABAINI, N. M.; GOMES-DA-COSTA, S. M. Produção de biomassa de cogumelo-do-sol e de shitake em resíduos agroindustriais. B. do CEPPA, v. 27, n. 2, p. 183-190, 2009.

26 MANZI, P.; PIZZOFERRATO, L. $\beta$-glucans in edible mushrooms. Food Chemistry, v. 68, n. 3, p. 315-318, 2000.

27 MIRA, G. S.; GRAF, H.; CÂNDIDO, L. M. B. Visão retrospectiva em fibras alimentares com ênfase em beta-glucanas no tratamento do diabetes. Brazilian Journal of Pharmaceutical Sciences, v. 45, n. 1, p. 11-20, 2009.

28 MIZUNO, T. The extraction and development of antitumor active-polysaccharides from medicinal mushrooms in Japan. Int. J. Med. Mushrooms, v. 1, p. 9-30, 1999.

29 MORAES, J. O.; PERTUZATTI, P. B.; CORREAA, F. V.; SALAS-MELLADO, M. L. M. Study of rabbiteye blueberry (Vaccinium ashei Reade) in the process of food products. Ciênc. Tecnol. Aliment., v. 27, supl., p. 18-22, 2007.

30 MOURÃO, F.; UMEO, S. H.; TAKEMURA, O. S.; LINDE, G. A.; COLAUTO, N. B. Antioxidant activity of Agaricus brasiliensis basidiocarps on different maturation phases. Braz. J. Microbiol., v. 42, n. 1, p. 197-202, 2011.

31 OHNO, N.; FURUKAWA, M.; MIURA, N. N.; ADACHI, Y.; MOTOI, M.; YADOMAE, T. Antitumor $\beta$-glucan from the cultured fruit body of Agaricus blazei. Biol. Pharm. Bull., v. 24, n. 7, p. 820-828, 2001.

32 PAPASPYRIDI, L.; ALIGIANNIS, N.; CHRISTAKOPOULOS, P.; SKALTSOUNIS, A.; FOKIALAKIS, N. Production of bioactive metabolites with pharmaceutical and nutraceutical interest by submerged fermentation of Pleurotus ostreatus in 
a batch stirred tank bioreactor. Procedia Food Science, v. 1, p. 1746-1752, 2011a.

33 PAPASPYRIDI, L. M.; KATAPODIS, P.; GONOU-ZAGOU, Z.; KAPSANAKI-GOTSI, E.; HRISTAKOPOULOS, P. Growth and biomass production with enhanced b-glucan and dietary fibre contents of Ganoderma australe ATHUM. 4345 in a batch-stirred tank bioreactor. Eng. Life Sci., v. 11, n. 1, p. 65-74, 2011 b.

34 PARK, Y. K.; IKEGAKI, M.; ALENCAR, S. M.; AGUIAR, C. L. Determinação da concentração de beta-glucano em cogumelo Agaricus blazei Murill por método enzimático. Ciênc. e Tecnol. Aliment., v. 23, n. 3, p. 312-316, 2003.

35 PRIETO, P.; PINEDA, M.; AGUILAR, M. Spectrophotometric quantitation of antioxidant capacity through the formation of a phosphomolybdenum complex: specific application to the determination of vitamin E. Analytical Biochemistry, v. 269, p. 337-341, 1999.

36 QUEIROZ, V. A. V.; CARNEIRO, H. L.; DELIZA, R.; RODRIGUES, J. A. S.; VASCONCELLOS, J. H.; TARDIN, F. D.; QUEIROZ, L. R. Genótipos de sorgo para produção de barra de cereais. Pesq. Agropec. Bras., v. 47, n. 2, p. 287-293, 2012.

37 ROBERTO, B. S. Resíduo de goiaba: metabolismo em ratos e aplicabilidade em barras de cereais. 2012. 163 f. Dissertação (Mestrado em Ciência e Tecnologia de Alimentos)-Universidade Federal de Santa Maria, Santa Maria, 2012.

38 ROCKENBACH, I. I.; SILVA, G. L.; RODRIGUES, E.; GONZAGA, L. V.; FETT, R. Atividade antioxidante de extratos de bagaço de uva das variedades Regente e Pinot Noir (Vitis vinifera). Rev. Inst. Adolfo Lutz, v. 66, n. 2, p. 158-163, 2007.

39 ROCKENBACH, I. I.; SILVA, G. L.; RODRIGUES, E.; KUSKOSKI, E. M.; FETT, R. Influência do solvente no conteúdo total de polifenóis, antocianinas e atividade antioxidante de extratos de bagaço de uva (Vitis vinifera) variedades Tannat e Ancelota. Ciênc. Tecnol. Aliment., v. 28, Supl., p. 238-244, 2008.

40 ROSLI, W. I. W.; AISHAH, M. S. Pleurotus sajor-caju (PSC) improves nutrient contents and maintains sensory properties of carbohydrate-based products. International Journal of Medical and Biological Sciences, v. 6, p. 156-158, 2012.

41 SAASTAMOINEN, M.; PLAAMI, S.; KUMPULAINEN, J. Beta-glucan and phytic acid content of oats cultivated in Finland. Acta Agriculturae Scandinavica, v. 42, n. 1, p. 6-11, 1992.

42 SANDULA, J.; KOGAN, G.; KACURAKOVA, M.; MACHOVA, E. Microbial (1-3)-beta-D-glucans, their preparation, physicochemical characterization and immunomodulatory activity. Carbohydrate Polymers, v. 38, n. 3, p. 247-253, 1999.

$43 \mathrm{SHU}, \mathrm{C} . ;$ LIN, K.; WEN, B. Effects of culture temperature on the production of bioactive polysaccharides by Agaricus blazei in batch cultures. J. Chem. Technol. Biotechnol., v. 82, p. 831-836, 2007.

44 SILVA, A. C.; JORGE, N. Antioxidant properties of Lentinus edodes and Agaricus blazei extracts. Journal of Food Quality, v. 34, p. 386-394, 2011.

45 SILVA, A. C.; OLIVEIRA, M. C.; DEL RE, P. V.; JORGE, N. Utilização de extrato de cogumelo como antioxidante natural em óleo vegetal. Ciênc. Agrotec., v.33, n.4, p. 1103-1108, 2009.

46 SINGLETON, V. L.; ROSSI, J. A. Colorimetry of total phenolics with phosphomolybdic phosphotungstic acid reagents. American Journal Enology Viticulture, v. 16, p. 144-158, 1965.

47 SOARES, A. A.; SOUZA, C. G. M.; DANIEL, F. M.; FERRARI, G. P.; COSTA, S. M. G.; PERALTA, R. M. Antioxidant activity and total phenolic content of Agaricus brasiliensis (Agaricus blazei Murril) in two stages of maturity. Food Chemistry, v. 112, p. 775-781, 2009.

48 SUN-WATERHOUSE, TEOH, A.; MASSAROTTO, C.; WIBISONO, R.; WADHWA, S. Comparative analysis of fruit-based functional snack bars. Food Chemistry, v. 119, p. 1369-1379, 2010.

49 VANNUCCHI, H.; MARCHINI, J.S. Nutrição e metabolismo: nutrição clínica. Rio de Janeiro: Guanabara Koogan, 2007. v. 1.

50 YADAV, D.; MASSOD, N.; LUQMAN, S.; BRINDHA, P.; GUPTA, M. M. Antioxidant furofuran lignans from Premna integrifolia. Industrial Crops and Products, v. 41, p. 397-402, 2013.

51 ZHANG, Z.; LV, G.; PAN, H.; FAN, L.; SOCCOL, C. R.; PANDEY, A. Antioxidants from wheat fermented by C. militaris. Food Technol. Biotechnol., v. 50, n. 1, p. 32-39, 2012.

\section{AGRADECIMENTOS}

Os autores agradecem ao PPGEAL/UFPR, à UNICENTRO e à CAPES. 